\title{
REVIEW
}

\section{Alzheimer's disease diagnostic criteria: practical applications}

\author{
Jeffrey Cummings*
}

\begin{abstract}
Alzheimer's disease (AD) can be identified prior to the occurrence of dementia by using biomarkers. Three phases of $A D$ are recognized: an asymptomatic biomarker-positive phase, a phase with positive biomarkers and mild cognitive deficits, and a dementia phase. Codification of these phases was first accomplished in 2007 by an International Work Group (IWG) led by Bruno Dubois. The definitions relevant to the approach were further clarified in 2010. In 2011, the National Institute on Aging/Alzheimer's Association (NIA/AA) established three work groups to develop definitions and criteria for these three phases of AD. The criteria of the IWG and those of the NIA/AA have many similarities and important differences. The two sets of criteria concur in recognizing the onset of $A D$ prior to dementia. The three phases of AD described in both sets of criteria embrace the same clinical entities but with different terminologies and emphases. IWG criteria emphasize a single clinico-biological approach that includes all symptomatic phases of $A D$ and uses the same diagnostic framework across the spectrum of symptomatic disease; the NIA/AA criteria apply different diagnostic approaches to the three phases. Biomarkers are an integrated and required part of the IWG criteria and are optional in the NIA/AA approach. Both sets of criteria have substantial strengths, but new information demonstrates shortcomings that can be addressed in future revisions of the criteria. These new criteria have profound implications, including greatly increasing the number of people identified as suffering from $A D$ and increasing the time that patients will spend with knowledge of the presence of the disease.
\end{abstract}

*Correspondence: cumminj@ccf.org

Cleveland Clinic Lou Ruvo Center for Brain Health, 888 W. Bonneville Avenue, Las Vegas, NV 89106, USA
Alzheimer's disease (AD) has traditionally been defined as a type of dementia, and criteria have been provided by the National Institute of Neurological and Communicative Disorders and Stroke - Alzheimer's Disease and Related Disorders Association (NINCDS-ADRDA) [1], the fourth edition of the Diagnostic and Statistical Manual of Mental Disorders (DSM-IV) [2], and the 10th revision of the International Classification of Diseases (ICD-10) [3]. Of these sets of criteria, the NINCDSADRDA criteria were most widely used in dementia research. There were several notable aspects of these criteria: (a) patients had to have cognitive deficits and functional compromise severe enough to meet criteria for dementia, (b) a confirmed diagnosis depended on postmortem examination, (c) the most accurate diagnosis that the clinician could make for the living patient was 'probable AD', (d) other possible causes of cognitive impairment had to be excluded by the clinician, and (e) the cognitive deficits were not operationalized for characteristics or severity. When applied by expert clinicians, these criteria have an $80 \%$ positive predictive value and a $60 \%$ negative predictive value for the accurate clinical diagnosis of $\mathrm{AD}$ when compared with postmortem examination [4].

Biomarker studies have forced a reconsideration of the time course of AD. Numerous studies of cerebrospinal fluid (CSF) demonstrate that amyloid beta protein (A $\beta$ ) declines and that total tau and phosphorylated tau levels increase several years before the onset of $\mathrm{AD}$ dementia $[5,6]$. Similarly, amyloid imaging studies demonstrate that amyloid is deposited in the brain several years prior to the onset of dementia in AD [6,7]. Atrophy on magnetic resonance imaging (MRI), hypometabolism on fluorodeoxyglucose (FDG) positron emission tomography (PET), and hypoperfusion on single-photon emission computed tomography (SPECT) all show abnormalities prior to the onset of dementia and coincident with or in anticipation of the onset of cognitive impairment [8-10]. These observations indicate that the presence of the pathological process characteristic of $\mathrm{AD}$ can be identified several years before the onset of $\mathrm{AD}$ dementia and that the dementia form of $\mathrm{AD}$ represents a late phase of the biological process. On the basis of data from biomarkers, 
$\mathrm{AD}$ can be reconceptualized as a progressive disorder that advances from biological changes in the brain with no accompanying cognitive abnormalities, to a state of memory impairment with biomarker abnormalities indicative of $\mathrm{AD}$, to mild, moderate, severe, and profound stages of AD dementia.

\section{International Work Group criteria}

Dubois and colleagues, of the International Work Group (IWG), developed new criteria for the diagnosis of AD as a clinico-biological entity [11] and produced a lexicon of terminology to address the complex issues associated with this marked revision of the way $\mathrm{AD}$ is conceived [12]. The author of this review is a member of the IWG. The new criteria have several important aspects: (a) the diagnosis of $\mathrm{AD}$ can be made in the living individual and is no longer dependent on autopsy confirmation; this is a shift from the clinico-pathological paradigm to a clinicobiological paradigm; (b) the diagnosis of $\mathrm{AD}$ can be made with greater certainty and does not use the terminology of 'probable AD'; (c) other diseases can be excluded with biomarkers, addressing the poor negative predictive value of earlier criteria; (d) the diagnosis of $\mathrm{AD}$ can be made prior to the onset of dementia; (e) the identification of $\mathrm{AD}$ pathology placing the person at risk for progressing to symptomatic $\mathrm{AD}$ can be made in asymptomatic individuals; (f) the same clinico-biological approach and same criteria can be used for diagnosis of all stages of symptomatic $\mathrm{AD}$ regardless of whether dementia is present; (g) the most common phenotype and typical presentation of $\mathrm{AD}$ are a hippocampal type of memory abnormality with poor storage and more response to cuing as demonstrated on a Free and Cued Selective Reminding Test; (h) several biomarkers can support the presence of $\mathrm{AD}$ pathology and fulfill the criteria; and (i) pathophysiologic (amyloid imaging and CSF $\mathrm{A} \beta$ and tau measures) and topographic (MRI, FDG PET, and SPECT) biomarkers are recognized [11,12]. In the IWG criteria, the concept of mild cognitive impairment (MCI) is abandoned in favor of the more specific prodromal $A D$ for those patients with symptomatic predementia AD. The IWG criteria do not offer any nomenclature for the general nonspecific syndrome of 'MCI' in favor of developing criteria that use biomarkers to identify that subset of $\mathrm{MCI}$ patients who are in the predementia phase of AD.

Patients without cognitive abnormalities and with positive biomarkers are identified as having AD pathology, an $\mathrm{AD}$ risk state. The IWG describes $\mathrm{AD}$ as a clinicobiological disorder; if no symptoms are present, then this is not a disease state. The presence of the biomarker represents a risk factor for progressing to $\mathrm{AD}$ in the future. The proportion of people who progress, the time frame for progression, and additional risk factors for progression are currently not fully defined [13-15]. The update of the criteria expanded the identification of different manifestations of AD dementia or atypical forms of $\mathrm{AD}$ dementia, including posterior cortical atrophy, the frontal variant of $\mathrm{AD}$ dementia, and the logopenic presentation of AD dementia [12].

Although these criteria represent a conceptual advance, they can be improved. Since the publication of these criteria, it has become evident that the physiological markers are more specific to the AD biological process; topographic markers are more sensitive to disease progression but less specific to AD [13]. Atrophy, hypometabolism, and hypoperfusion occur in a variety of disease states, whereas amyloid abnormalities are present in a very restricted number of disorders, predominantly $\mathrm{AD}$. Moreover, the absence of $\mathrm{A} \beta$ abnormalities is strong evidence against $\mathrm{AD}$ as the cause of the cognitive changes, whereas negative findings on MRI, FDG PET, or SPECT do not have the same negative predictive value.

Requiring a pathophysiologic biomarker to support the presence of $\mathrm{AD}$ will improve diagnostic accuracy. In addition, the IWG criteria recognize atypical forms of $\mathrm{AD}$ dementia but not atypical manifestations of prodromal AD. Extending this diagnostic framework backwards into prodromal AD will improve diagnostic continuity and sensitivity. The issue of mutation carriers can also be clarified in the criteria. The presence of a hippocampal type of amnestic disorder in a patient who has a known causative mutation and who presents at an age compatible with the mutation expression can be diagnosed as prodromal $\mathrm{AD}$ or $\mathrm{AD}$ dementia depending on the presence of the accompanying functional deficits. Merely being in a family known to carry the mutation, however, should not be sufficient to make this diagnosis, as there are unusual non-AD causes of hippocampal amnesia and these can occur in non-carriers of mutationbearing families. Revisiting and adjusting the criteria in this way would reduce the opportunity for diagnostic ambiguity in their application.

\section{National Institute on Aging/Alzheimer's Association criteria}

After the two publications of the IWG criteria, the National Institute on Aging (NIA) and the Alzheimer's Association (AA) convened three working groups to develop criteria for the asymptomatic, minimally symptomatic, and dementia phases of AD [16-18]. These criteria share many features with the IWG criteria, including recognition of an asymptomatic biomarker-positive phase of AD pathology, identification of a predementia symptomatic phase of $\mathrm{AD}$, retention of criteria for $\mathrm{AD}$ dementia, integration of biomarkers into some approaches to the diagnostic process, and categorization of biomarkers into two types: one identifying $A \beta$ abnormalities and one for neurodegeneration. 
A notable aspect of the NIA/AA criteria for the preclinical asymptomatic phase of $\mathrm{AD}$ is the description of a sequential appearance of abnormalities from stage 1 with abnormalities of amyloid only, to stage 2 with biomarkers of both amyloid abnormalities and neurodegeneration, to stage 3 with both types of biomarkers and minimal clinical decline not meeting criteria for MCI [16].

The predementia symptomatic phase of $\mathrm{AD}$ retains the concept of MCI in the NIA/AA approach [17]. This has the advantage of being able to be implemented in clinical practice where biomarkers are unavailable. Non-AD as well as AD types of $\mathrm{MCI}$ are recognized in the criteria. There is some ambiguity in the use of 'MCI' versus 'MCI due to $\mathrm{AD}$ ' in the criteria. $\mathrm{MCI}$ is defined by using the original criteria of Petersen and colleagues [19]. Biomarkers are not required for the diagnosis of $\mathrm{MCI}$, although the diagnosis of MCI due to $\mathrm{AD}$ would require biomarker support given the heterogeneous nature of the MCI syndrome [20,21]. For research application, biomarkers are introduced and stratified. If biomarkers of both amyloid abnormalities and neurodegeneration are present, the term 'MCI due to AD - high likelihood' is applied; if only one type of biomarker is collected and is positive, then the term 'MCI due to $\mathrm{AD}$ - intermediate likelihood' is used. If no biomarker is collected, the results are ambiguous, or two biomarkers are contradictory, then the biomarkers are uninformative and the relationship to AD is uncertain. The NIA/AA $\mathrm{MCI}$ approach does not establish a continuity with the criteria suggested for preclinical AD in which both types of biomarkers are implied to be positive by the time the patient becomes symptomatic [16].

The stratification of biomarkers offered in the NIA/AA approach is challenging to apply in practice since biomarkers are not strictly and consistently related to each other. Patients with MCI and positive amyloid imaging but with an MRI that does not reach a predefined cutoff for excessive atrophy would receive a diagnosis of MCI due to $\mathrm{AD}$ of uncertain likelihood since the biomarkers are inconsistent with each other, although there are few competing diagnoses for this set of observations. Likewise, a patient with $\mathrm{MCI}$ and hippocampal atrophy on MRI and negative amyloid imaging would receive the same diagnosis of $\mathrm{MCI}$ due to $\mathrm{AD}$ of uncertain likelihood, although the diagnosis of $\mathrm{AD}$ in this case would be biologically unlikely.

$\mathrm{MCI}$ is distinguished from dementia by the presence of abnormalities of activities of daily living (ADLs). This is an arbitrary distinction based on clinical judgment and is subject to both clinician biases and patient or caregiver reporting errors. Studies of ADL rating scales in MCI demonstrate that impairment of at least some high-level instrumental ADLs is common in MCI [22]. Morris [23] analyzed the effect of the NIA/AA criteria on diagnostic divisions. The author showed that, if these criteria were applied, many conditions currently classified as mild AD would be identified as MCI. This shift would have many ramifications, including allowing patients with a more advanced pathology into MCI trials.

The NIA/AA criteria for AD dementia retain most of the features of the past diagnosis of probable AD [1] despite the limited positive predictive value and poor negative predictive value of these criteria [4]. Diagnostic standards for all-cause dementia are provided, and 10 categories of dementia of the $\mathrm{AD}$ type - including probable $\mathrm{AD}$ dementia, possible $\mathrm{AD}$ dementia, probable or possible $\mathrm{AD}$ dementia with evidence of the $\mathrm{AD}$ pathophysiological process, and pathophysiologically proved AD dementia - are established [18]. Probable AD dementia meets clinical diagnostic criteria without supporting biomarker evidence of $\mathrm{AD}$. Biomarkers are integrated into the criteria to describe probable or possible $\mathrm{AD}$ dementia with pathophysiological evidence of $\mathrm{AD}$ and are stratified as of high likelihood of being due to $\mathrm{AD}$ if amyloid abnormalities and evidence of neurodegeneration are both present and of intermediate likelihood of being due to $\mathrm{AD}$ if only one type of biomarker evidence is present.

The terminology of 'probable AD dementia of intermediate likelihood of being due to AD' may be confusing for clinical and research application. When NIA/AA criteria are used, the patient with the clinical syndrome of probable $\mathrm{AD}$, no available structural imaging, and positive amyloid imaging would be receive a diagnosis of probable AD of intermediate likelihood of being due to $\mathrm{AD}$, although there are few (if any) alternatives to the diagnosis of $\mathrm{AD}$ in this case. A patient with an AD-like phenotype, atrophy on MRI, and negative amyloid imaging would receive a diagnosis of probable $\mathrm{AD}$ with uncertain likelihood of $\mathrm{AD}$, although this person is unlikely to have $\mathrm{AD}$. The person with the $\mathrm{AD}$ dementia phenotype and no biomarkers would receive a diagnosis of probable AD.

The NIA/AA criteria are an improvement over the preceding diagnostic approach of the NINCDS/ADRDA criteria and are a step toward electively integrating new biomarker information into a diagnostic framework. Like the IWG criteria, the NIA/AA criteria can be improved. The use of three different approaches to diagnose the three phases of the same illness fails to capture the evolving idea of a single disorder occurring along a spectrum of severity. The concept of a single stereotyped sequence of biomarker and clinical progression in the preclinical phase of AD does not reflect the heterogeneity of findings observed in clinical practice [24]. The ambiguity of $\mathrm{MCI}$ versus $\mathrm{MCI}$ due to $\mathrm{AD}$ can be clarified, and the diagnostic complexity of the approach to $\mathrm{AD}$ dementia offered can be simplified to assist in clinical 
application. The criteria for identifying someone as manifesting $\mathrm{MCI}$ rather than mild $\mathrm{AD}$ may not be sufficiently stringent [23]. The stratification of biomarkers used in both the MCI and AD dementia approaches is challenging in clinical implementation; the data support the diagnostic specificity of amyloid biomarkers over biomarkers of neurodegeneration and these observations can be incorporated into improved criteria.

\section{Implications of new criteria}

Two pivotal reconceptualizations of AD have occurred in the history of our understanding of this disease, and both have led to dramatic increases in the recognition of the number of people affected. The first was led by Robert Katzman, who in 1976 [25] drew attention to the identity between 'presenile' AD and 'senile dementia. He recognized that $A D$ is the major cause of progressive cognitive impairment in the elderly and is bound to grow to epidemic proportions with the aging of the world's population. The second is the pioneering work of the IWG. This work synthesizes the available evidence into a cohesive framework for recognition of $\mathrm{AD}$ as a clinicobiological disorder that can be diagnosed in life and embraces a spectrum of severity from very mild symptoms to profound dementia [11,12]. This change in paradigm also has the effect of greatly increasing the number of individuals recognized as manifesting $\mathrm{AD}$. The projection of 80 million cases of AD by 2040 [26] is based on projections of $\mathrm{AD}$ dementia and will be multiplied by a factor of at least 2 if predementia cases of $\mathrm{AD}$ are included.

Another effect of making the diagnosis earlier in the disease and including predementia cases in the diagnosis of $\mathrm{AD}$ is the dilemma it raises for patient care [27]. Patients deserve to know what the clinician knows and to be informed about the limits of our knowledge. The diagnosis of prodromal AD is often - but not always associated with transition to $\mathrm{AD}$ dementia over the ensuing few years. Hertze and colleagues [28] found that $71 \%$ of patients with the syndrome of MCI and AD-type CSF findings, including low $A \beta$ and high tau, progressed to AD dementia within 5 years. Twenty-nine percent of such patients did not develop a dementia syndrome and were cognitively stable even after half a decade of observation [28]. This represents uncertainty for the physician and hope for the patient and patient's family. When individuals are given a diagnosis of prodromal AD, they will carry the diagnosis of $\mathrm{AD}$ for many years and must be resigned to the uncertainty of the duration of this phase of the disorder. Helping patients live with AD will be a major theme of coming research and care.

\section{Comment}

Rapidly evolving information about the biology of AD and biomarker windows on the disorder are reshaping our understanding of the disease at an accelerating pace. The IWG and the NIA/AA work groups support the diagnosis of AD prior to the onset of dementia and point to how best to integrate biomarkers into diagnostic criteria. Both sets of available criteria can be revised to better capture current data on the role of amyloid as a diagnostic hallmark and early indicator of the presence of the AD.

An important goal of developing new criteria is to support clinical trials and advance the new therapeutics for AD. So that optimal cognitive function is maintained and the progression of $\mathrm{AD}$ into the dementia phase is deferred, a diagnostic framework for predementia AD is needed. Many trials have involved patients with diagnosed MCI and none has led to the development of a new treatment or extension of currently available treatments into the MCI phase of $\mathrm{AD}$. The heterogeneity of $\mathrm{MCI}$ and the lack of uniform biological targets in this nonspecific syndrome may have contributed to these failures [20,21]. IWG criteria for prodromal AD have been successfully implemented in current phase 2 clinical trials for small molecules, immunotherapies, and medical foods and have been accepted by the European Medicines Agency $[29,30]$ for use in AD clinical trials. A conundrum in the clinical trial application of research diagnostic criteria is how to make them specific enough to capture a population with a homogeneous biology but not so narrow that they exclude many patients who have AD but who lack a specific phenotype or biomarker profile.

The preclinical phase of AD (NIA/AA criteria) or $\mathrm{AD}$ risk state (IWG criteria) sets the stage for the implementation of prevention trials of individuals who do not have symptoms of $\mathrm{AD}$ but who harbor the amyloid changes that have been associated with the presence of the disease. The goal of these studies will be to delay the onset of cognitive and functional decline. Such studies will be facilitated by refined diagnostic criteria.

The emergence of biomarkers is the basis for both justifying and formulating new diagnostic criteria. Without progress in biomarkers, there would be no need for new criteria. The IWG criteria require both clinical and biomarker evidence to identify the different phases of $\mathrm{AD}$ and $\mathrm{AD}$ pathology. In NIA/AA criteria, use of biomarkers is optional and clinical information alone can suffice for diagnosis of MCI or probable AD dementia. Among biomarkers, amyloid abnormalities may have a particularly informative role in $\mathrm{AD}$ risk states and prodromal AD.

Regulatory language used with the recent approval of florbetapir emphasizes that negative amyloid imaging in persons with cognitive impairment is inconsistent with identifying neuritic plaques as the cause of the cognitive decline and therefore inconsistent with a diagnosis of $\mathrm{AD}$ as the cause of the cognitive syndrome. $\mathrm{AD}$ is not 
currently excluded with negative amyloid imaging in either set of diagnostic criteria. It will be important to align regulatory and clinical criteria in future versions of these diagnostic approaches.

Positive amyloid imaging is less informative than negative imaging and occurs in patients with AD, Lewy body dementia of the AD type, or amyloid angiopathy and in cognitively normal persons $[31,32]$. The combination of careful description of the phenotype in conjunction with the use of biomarkers is critical to accurate diagnosis and will be assisted by diagnostic criteria.

Both sets of criteria emphasize the research nature and need for studies of the sensitivity, specificity, and positive and negative predictive value of the criteria. The urgent need of clinicians for guidance in how to apply biomarkers and improve diagnostic standards means that new technologies and approaches will be incorporated rapidly into clinical practice where the appropriate biomarkers are available. Rapidly advancing research on biomarkers provides new information with which to revise the criteria and enhance their clinical utility. This is a necessary step toward advancing new and desperately needed treatments for AD.

\section{Abbreviations}

$A \beta$, amyloid beta protein; AA, Alzheimer's Association; AD, Alzheimer's disease; $A D L$, activity of daily living; CSF, cerebrospinal fluid; FDG, fluorodeoxyglucose; IWG, International Work Group; MCI, mild cognitive impairment; MRI, magnetic resonance imaging; NIA, National Institute on Aging; NINCDS-ADRDA, National Institute of Neurological and Communicative Disorders and Stroke Alzheimer's Disease and Related Disorders Association; PET, positron emission tomography; SPECT, single-photon emission computed tomography.

\section{Competing interests}

The author has served as a consultant to the following companies: Abbott (Abbott Park, LL, USA), Acadia (San Diego, CA, USA), Adamas (Emeryville, CA USA), Anavex (Vancouver, BC, Canada), Astellas (Tokyo, Japan), Avanir (Aliso Viejo, CA, USA), Avid (Philadelphia, PA, USA), Baxter (Deerfield, IL, USA), Bayer (Emeryville, CA, USA), Bristol-Myers Squibb Company (Princeton, NJ, USA), Eisai (Woodcliff Lake, NJ, USA), Élan (Dublin, Ireland), EnVivo (Watertown, MA, USA), Forest (New York, NY, USA), General Electric (Fairfield, CT, USA), Genentech (South San Francisco, CA, USA), GlaxoSmithKline (Uxbridge, Middlesex, UK), Janssen (Neuss, Germany), Eli Lilly and Company (Indianapolis, IN, USA), Lundbeck (Copenhagen, Denmark), MedAvante (Hamilton, NJ, USA), Merck (Darmstadt, Germany), Neuronix (Yoqnea'm, Israel), Neurotrax (Bellaire, TX, USA), Novartis (Basel, Switzerland), Otsuka (Tokyo, Japan), Pfizer Inc (New York, NY, USA), Prana (Parkville, VIC, Australia), QR Pharma (Berwyn, PA, USA), Sanofi (Paris, France), Sonexa (San Diego, CA, USA), Takeda (Deerfield, IL, USA), and United BioSource Corporation (Chevy Chase, MD, USA). He owns stock or has stock options in ADAMAS, Prana, Sonexa, MedAvante, Neurotrax, Neurokos (Palo Alto, CA, USA), and QR Pharma. He has served as a speaker/lecturer for Eisai, Forest, Janssen, Novartis, Pfizer Inc, and Lundbeck. He owns the copyright of the Neuropsychiatric Inventory and has provided expert witness consultation regarding olanzapine and ropinerole.

Published: 5 September 2012

\section{References}

1. McKhann G, Drachman D, Folstein M, Katzman R, Price D, Stadlan EM: Clinical diagnosis of Alzheimer's disease: report of the NINCDS-ADRDA Work Group under the auspices of Department of Health and Human Services Task Force on Alzheimer's Disease. Neurology 1984, 34:939-944.

2. Diagnostic and Statistical Manual of Mental Disorders. 4th edition. Washington, DC: American Psychiatric Association; 1990.
3. International Classification of Diseases. 10th revision. Geneva, Switzerland: World Health Organization; 1992.

4. Beach TG, Monsell SE, Phillips LE, Kukull W: Accuracy of the clinical diagnosis of Alzheimer disease at National Institute on Aging Alzheimer Disease Centers, 2005-2010. J Neuropathol Exp Neuro 2012, 71:266-273.

5. Buchhave P, Minthon L, Zetterberg H, Wallin AK, Blennow K, Hansson O: Cerebrospinal fluid levels of $\beta$-amyloid $1-42$, but not of tau, are fully changed already 5 to 10 years before the onset of Alzheimer dementia. Arch Gen Psychiatry 2012, 69:98-106.

6. Fagan AM, Mintun MA, Shah AR, Aldea P, Roe CM, Mach RH, Marcus D, Morris JC, Holtzman DM: Cerebrospinal fluid tau and ptau(181) increase with cortical amyloid deposition in cognitively normal individuals: implications for future clinical trials of Alzheimer's disease. EMBO Mol Med 2009, 1:371-380.

7. Aizenstein HJ, Nebes RD, Saxton JA, Price JC, Mathis CA, Tsopelas ND, Ziolko SK, James JA, Snitz BE, Houck PR, Bi W, Cohen AD, Lopresti BJ, DeKosky ST, Halligan EM, Klunk WE: Frequent amyloid deposition without significant cognitive impairment among the elderly. Arch Neurol 2008, 65:1509-1517.

8. Westman E, Muehlboeck JS, Simmons A: Combining MRI and CSF measures for classification of Alzheimer's disease and prediction of mild cognitive impairment conversion. Neuroimage 2012, 62:229-238.

9. Ossenkoppele R, Tolboom N, Foster-Dingley JC, Adriaanse SF, Boellaard R, Yaqub M, Windhorst AD, Barkhof F, Lammertsma AA, Scheltens P, van der Flier $W M$, van Berckel BN: Longitudinal imaging of Alzheimer pathology using [(11)C]PIB, [ (18)F]FDDNP and [ (18)F]FDG PET. Eur J Nucl Med Mol Imaging 2012, 39:990-1000

10. Alegret $M$, Cuberas-Borrós $G$, Vinyes-Junqué $G$, Espinosa A, Valero S, Hernández I, Roca I, Ruíz A, Rosende-Roca M, Mauleón A, Becker JT, CastellConesa J, Tárraga L, Boada M: A two-year follow-up of cognitive deficits and brain perfusion in mild cognitive impairment and mild Alzheimer's disease. J Alzheimers Dis 2012, 30:109-120.

11. Dubois B, Feldman HH, Jacova C, Dekosky ST, Barberger-Gateau P, Cummings J, Delacourte A, Galasko D, Gauthier S, Jicha G, Meguro K, O'brien J, Pasquier F, Robert P, Rossor M, Salloway S, Stern Y, Visser PJ, Scheltens P: Research criteria for the diagnosis of Alzheimer's disease: revising the NINCDS-ADRDA criteria. Lancet Neurol 2007, 6:734-746. Review.

12. Dubois B, Feldman HH, Jacova C, Cummings JL, Dekosky ST, BarbergerGateau P, Delacourte A, Frisoni G, Fox NC, Galasko D, Gauthier S, Hampel H, Jicha GA, Meguro K, O'Brien J, Pasquier F, Robert P, Rossor M, Salloway S, Sarazin M, de Souza LC, Stern Y, Visser PJ, Scheltens P: Revising the definition of Alzheimer's disease: a new lexicon. Lancet Neurol 2010, 9:1118-1127.

13. van Rossum IA, Visser PJ, Knol DL, van der Flier WM, Teunissen CE, Barkhof F, Blankenstein MA, Scheltens P: Injury markers but not amyloid markers are associated with rapid progression from mild cognitive impairment to dementia in Alzheimer's disease. J Alzheimers Dis 2012, 29:319-327.

14. Heister D, Brewer JB, Magda S, Blennow K, McEvoy LK; Alzheimer's Disease Neuroimaging Initiative: Predicting $\mathrm{MCl}$ outcome with clinically available MRI and CSF biomarkers. Neurology 2011, 77:1619-1628.

15. Gomar JJ, Bobes-Bascaran MT, Conejero-Goldberg C, Davies P, Goldberg TE; Alzheimer's Disease Neuroimaging Initiative: Utility of combinations of biomarkers, cognitive markers, and risk factors to predict conversion from mild cognitive impairment to Alzheimer disease in patients in the Alzheimer's disease neuroimaging initiative. Arch Gen Psychiatry 2011, 68:961-969.

16. Sperling RA, Aisen PS, Beckett LA, Bennett DA, Craft S, Fagan AM, Iwatsubo T, Jack CR Jr., Kaye J, Montine TJ, Park DC, Reiman EM, Rowe CC, Siemers E, Stern Y, Yaffe K, Carrillo MC, Thies B, Morrison-Bogorad M, Wagster MV, Phelps CH: Toward defining the preclinical stages of Alzheimer's disease: recommendations from the National Institute on Aging-Alzheimer's Association workgroups on diagnostic guidelines for Alzheimer's disease. Alzheimers Dement 2011, 7:280-292.

17. Albert MS, DeKosky ST, Dickson D, Dubois B, Feldman HH, Fox NC, Gamst A, Holtzman DM, Jagust WJ, Petersen RC, Snyder PJ, Carrillo MC, Thies B, Phelps $\mathrm{CH}$ : The diagnosis of mild cognitive impairment due to Alzheimer's disease: recommendations from the National Institute on AgingAlzheimer's Association workgroups on diagnostic guidelines for Alzheimer's disease. Alzheimers Dement 2011, 7:270-279.

18. McKhann GM, Knopman DS, Chertkow H, Hyman BT, Jack CR Jr., Kawas CH, Klunk WE, Koroshetz WJ, Manly JJ, Mayeux R, Mohs RC, Morris JC, Rossor MN, Scheltens P, Carrillo MC, Thies B, Weintraub S, Phelps CH: The diagnosis of dementia due to Alzheimer's disease: recommendations from the 
National Institute on Aging-Alzheimer's Association workgroups on diagnostic guidelines for Alzheimer's disease. Alzheimers Dement 2011, 7:263-269.

19. Petersen RC, Smith GE, Waring SC, Ivnik RJ, Tangalos EG, Kokmen E: Mild cognitive impairment: clinical characterization and outcome. Arch Neurol 1999, 56:303-308. Erratum in: Arch Neurol 1999, 56:760.

20. Jicha GA, Parisi JE, Dickson DW, Johnson K, Cha R, Ivnik RJ, Tangalos EG, Boeve BF, Knopman DS, Braak H, Petersen RC: Neuropathologic outcome of mild cognitive impairment following progression to clinical dementia. Arch Neurol 2006, 63:674-681.

21. Ganguli M, Snitz BE, Saxton JA, Chang CC, Lee CW, Vander Bilt J, Hughes TF, Loewenstein DA, Unverzagt FW, Petersen RC: Outcomes of mild cognitive impairment by definition: a population study. Arch Neurol 2011, 68:761-767.

22. Teng E, Becker BW, Woo E, Cummings JL, Lu PH: Subtle deficits in instrumental activities of daily living in subtypes of mild cognitive impairment. Dement Geriatr Cogn Disord 2010, 30:189-197.

23. Storandt M, Head D, Fagan AM, Holtzman DM, Morris JC: Toward a multifactorial model of Alzheimer disease. Neurobiol Aging 2012, 33:2262-2271

24. Morris JC: Revised criteria for mild cognitive impairment may compromise the diagnosis of Alzheimer disease dementia. Arch Neurol 2012, Feb 6. [Epub ahead of print].

25. Katzman R: Editorial: The prevalence and malignancy of Alzheimer disease. A major killer. Arch Neurol 1976, 33:217-218.

26. Ferri CP, Prince M, Brayne C, Brodaty H, Fratiglioni L, Ganguli M, Hall K, Hasegawa K, Hendrie H, Huang Y, Jorm A, Mathers C, Menezes PR, Rimmer E, Scazufca M; Alzheimer's Disease International: Global prevalence of dementia: a Delphi consensus study. Lancet 2005, 366:2112-2117.
27. Karlawish J: Addressing the ethical, policy and social challenges of preclinical Alzheimer's disease. Neurology 2011, 77:1487-1493.

28. Hertze J, Minthon L, Zetterberg H, Vanmechelen E, Blennow K, Hansson O: Evaluation of CSF biomarkers as predictors of Alzheimer's disease: a clinical follow-up study of 4.7 years. J Alzheimers Dis 2010, 21:1119-1128.

29. Isaac M, Vamvakas S, Abadie E, Jonsson B, Gispen C, Pani L: Qualification opinion of novel methodologies in the predementia stage of Alzheimer's disease: cerebrospinal fluid related biomarkers for drug affecting amyloid burden - regulatory considerations by European Medicines Agence focusing in improving benefit/risks in regulatory trials. European Neuropsychopharm 2011, 21:781-788.

30. Truffinet $P$, Bordet $R$, Ménard J: Relevance of the evaluation criteria used in clinical trials for Alzheimer's disease. Therapie 2009, 64:139-148.

31. Villemagne VL, Ong K, Mulligan RS, Holl G, Pejoska S, Jones G, O'Keefe G, Ackerman U, Tochon-Danguy H, Chan JG, Reininger CB, Fels L, Putz B, Rohde $B$, Masters CL, Rowe CC: Amyloid imaging with (18)F-florbetaben in Alzheimer disease and other dementias. J Nucl Med 2011, 52:1210-1217.

32. Johnson KA, Gregas M, Becker JA, Kinnecom C, Salat DH, Moran EK, Smith EE, Rosand J, Rentz DM, Klunk WE, Mathis CA, Price JC, Dekosky ST, Fischman AJ, Greenberg SM: Imaging of amyloid burden and distribution in cerebral amyloid angiopathy. Ann Neurol 2007, 62:229-234.

\section{doi:10.1186/alzrt138}

Cite this article as: Cummings J: Alzheimer's disease diagnostic criteria: practical applications. Alzheimer's Research \& Therapy 2012, 4:35. 